\title{
Epithelial membrane protein 3 functions as an oncogene and is regulated by microRNA-765 in primary breast carcinoma
}

\author{
XIAO CHUN HONG ${ }^{1}$, YUAN JIAN FEN ${ }^{2}$, GUO CHUN YAN ${ }^{2}$, HONG HONG $^{2}$, \\ CAO HONG YAN ${ }^{2}$, LI WEI BING ${ }^{2}$ and YU HAI ZHONG ${ }^{2}$ \\ ${ }^{1}$ Department of Clinical Laboratory, Nantong Cancer Hospital; ${ }^{2}$ Department of Clinical Laboratory, \\ Traditional Chinese Medicine Hospital, Nantong, Jiangsu 226001, P.R. China
}

Received September 14, 2014; Accepted July 10, 2015

DOI: $10.3892 / \mathrm{mmr} .2015 .4326$

\begin{abstract}
Epithelial membrane protein 3 (EMP3) is a transmembrane signaling molecule, which is important in the regulation of apoptosis, differentiation and invasion of cancer cells. However, the specific function and regulatory mechanism of EMP3 in primary breast carcinoma remain to be elucidated. In the present study, the mRNA and protein levels of EMP3 were observed to be upregulated in primary breast carcinoma tissues, compared with normal tissues. It was hypothesized that the overexpression of EMP3 was correlated with the downregulation of microRNA-765 (miR-765), an underexpressed miRNA in primary breast carcinoma tissues. Functional analysis demonstrated that EMP3 was regulated by miR-765 through binding to its 3'untranslated region. In addition, the knockdown of EMP3 and miR-765 had similar effects on the inhibition of proliferation and invasion in SK-BR-3 cells. These results provided novel insight into the regulatory mechanism of EMP3 in primary breast carcinoma.
\end{abstract}

\section{Introduction}

Epithelial membrane protein 3 (EMP3) belongs to the peripheral myelin protein 22-kDa (PMP22) gene family of small hydrophobic membrane glycoproteins, which include four closely associated members; PMP22, EMP1, EMP2 and EMP3, in additional to the more distant member, MP20 $(1,2)$. The EMP3 gene, which encodes EMP3, is located on the 19q13.3 band (3) and it has high levels of transcriptional expression in a broad range of tissues (2). Previous studies have reported that EMP3 may be involved in regulating cell proliferation and apoptosis (1). In addition, studies have demonstrated that the overexpression of EMP3 is positively associated with the

Correspondence to: Dr Yu Hai Zhong, Department of Clinical Laboratory, Traditional Chinese Medicine Hospital, 41 Jianshe Road, Nantong, Jiangsu 226001, P.R. China

E-mail: yuhaizhong911@163.com

Key words: epithelial membrane protein 3, microRNA-765, cell proliferation, cell invasion, primary breast carcinoma invasive status of human mammary carcinoma cell lines (4-6). However, its regulatory mechanism remains to be fully elucidated.

MicroRNAs (miRNAs) are small, noncoding, endogenous single RNA molecules, which are important in gene expression via directly binding to the 3'-untranslated region (3'UTR) of target gene mRNA, resulting in mRNA cleavage or translational repression (7). miRNAs are differentially expressed in human cancer and are essential in carcinogenesis, including primary breast carcinoma. Previous studies have reported, using microarray profiles, that numerous miRNAs are dysregulated in breast carcinoma, including miRNA-451 (miR-451), miR-155 and miR-21 $(8,9)$. However, few studies have investigated the association between miRNA and EMP3 in primary breast carcinoma.

The aim of the present study was to investigate the expression levels of EMP3 in primary breast tissues, compared with adjacent normal tissues. Subsequently, the association between EMP3 and miRNAs in the development of primary breast carcinoma was examined. To further confirm the role of miRNA in regulation of the expression of EMP3, short hairpin (sh)RNAs were designed in order to knock down EMP3 in breast cancer cells. The results of these investigations aim to provide evidence that miRNAs are important in the regulation of EMP3 in primary breast carcinoma tumorigenesis, to provide novel insight into the mechanism of EMP3 regulation in primary breast carcinoma.

\section{Materials and methods}

Clinical primary breast carcinoma specimen and RNA isolation. A total of 6 paired samples of primary breast carcinoma and corresponding adjacent normal tissues were obtained at the Nantong Cancer Hospital (Nantong, China) from patients, following the provision of informed consent. Ethical approval was granted by the Ethics Committee of Nantong Cancer Hospital (Nantong, China). The total RNA was extracted from the tissues using TRIzol reagent (Invitrogen Life Technologies, Carlsbad, CA, USA), according to the manufacturer's instructions. The average age of the six female patients was $57.0 \pm 7.2$ years. According to the tumor-node-metastasis classification, five patients wee diagnosed as stage I, and one patient was diagnosed as stage II. 
Cell culture and transfection. SK-BR-3 (HTB-30 ${ }^{\mathrm{TM}}$; American Type Culture Collection, Manassas, VA, USA) cells were cultured in Dulbecco's modified Eagle's medium, supplemented with $10 \%$ fetal bovine serum (FBS), $100 \mathrm{U} / \mathrm{ml}$ penicillin and $100 \mu \mathrm{g} / \mathrm{ml}$ streptomycin (Gibco Life Technologies, Grand Island, NY, USA). MDA-MB-231, MDA-MB-453 and MCF-7 were also purchased from the American Type Culture Collection. The SK-BR-3 cells were incubated at $37^{\circ} \mathrm{C}$ in a humidified chamber supplemented with $5 \% \mathrm{CO}_{2}$. The miRNA-765 mimic and negative control (control) were purchased from Guangzhou RiboBio, Co., Ltd. (Guangzhou, China). The cells were cultured to $30-50 \%$ confluence and transfected with $100 \mathrm{nM}$ of either the miR-765 mimic or negative control using Lipofectamine 2000 in Opti-MEM (Invitrogen Life Technologies), according to the manufacturer's instructions.

Cell growth assay. The cells were seeded in 96-well plates at a density of 8,000 cells/well and were transfected the following day. An MTT assay was used to determine the relative growth of the cells 12, 24, 36 and $48 \mathrm{~h}$ following transfection. MTT solution (20 $\mu \mathrm{l}$; Sigma-Aldrich, St. Louis, MO, USA) was added into $100 \mu \mathrm{l}$ culture media, and the cells were incubated for another $4 \mathrm{~h}$ at $37^{\circ} \mathrm{C}$. The optical density was then measured at $490 \mathrm{~nm}$ using a microplate reader (DR-200Bs; Diatek, Wuxi, China).

Reverse transcription-quantitative polymerase chain reaction $(R T-q P C R)$. RNA was extracted using TRIzol reagent (Invitrogen Life Technologies). The stem-loop qPCR method was used to detect the expression levels of miR-765. The detection of EMP3 mRNA levels was performed using RT-qPCR. The primer pairs for EMP3 were Forward 5'-CGAGAATGG CTGGCTGAAG-3' and reverse 5'-GCCACGCTGGTGCAA AG-3'.

The primers, which were used for miR-765 were, as described previously (10). All primers were purchased from Shanghai GenePharma (Shanghai, China). A SYBR Green Mix Taq ${ }^{\mathrm{TM}}$ kit (Takara Bio, Inc., Otsu, Japan) was used to trace the amplified DNA.

Western blot analysis. The protein concentration was measured using a bicinchoninic acid method (Sigma-Aldrich), following which $20 \mu \mathrm{g}$ protein was loaded onto gels for $10 \%$ SDS-PAGE for analysis. The separated proteins were transferred onto membranes (nitrocellulose membrane; Sigma-Aldrich) The primary antibodies used were rabbit polyclonal anti-human EMP3 antibody (1:1,000; cat. no. 73151; Abcam, Cambridge, MA, USA) and rabbit monoclonal anti-human actin antibody (1:1,000; cat. no. ab1801; Abcam), which were applied for $24 \mathrm{~h}$ at $4^{\circ} \mathrm{C}$. The secondary antibody used was goat anti-rabbit IgG antibody conjugated with horseradish peroxidase (1:1,000; cat. no. ab6721; Abcam).

Luciferase reporter assay and construction. Plasmids containing EMP3-3'UTR and EMP3-3'UTR mutation were constructed with technical support from Guangzhou Zhiyou Biotech Co., Ltd. (Guangdong, China). The 3'-UTR sequence of EMP3, predicted using miRwalk (http://www.umm. uni-heidelberg.de/apps/zmf/mirwalk/) to interact with miR-765 and a mutated sequence of the 3'-UTR sequence, was inserted into pGL3 vectors (Promega Corporation, Madison, WI, USA). Subsequent to transfection with miR-765 for $24 \mathrm{~h}$, the SK-BR-3 cells were transfected with the pGL3/EMP3-3'UTR and pGL3/EMP3-3'UTR mutant plasmids. The luciferase activity was measured 48 or $96 \mathrm{~h}$ after transfection using a Dual-Luciferase reporter assay system (Promega Corporation). The EMP3 shRNA plasmid was purchased from Santa Cruz Biotechnology, Inc. (cat. no. sc-97634-SH; Dallas, TX, USA).

Transwell assay. The invasion and migration of the cells was evaluated by assessing the ability of cells to pass through a Matrigel-coated membrane matrix (BD Biosciences, Franklin Lakes, CA, USA). The cells were seeded onto a Matrigel-coated membrane matrix, placed in the insert of a 24-well culture plate, $24 \mathrm{~h}$ after transfection. FBS was added to the lower chamber as a chemoattractant. After $24 \mathrm{~h}$, the invasive cells located on the lower surface of the chamber were stained with $0.1 \%$ crystal violet (Sigma-Aldrich) and were counted.

Statistical analysis. All data are presented as the mean \pm standard deviation of three independent experiments. Statistical analyses were performed using SPSS software, version 16.0 (SPSS, Inc., Chicago, IL, USA) and statistical significance between treatment and control groups was analyzed using Student's t-test. $\mathrm{P}<0.05$ was considered to indicate a statistically significant difference.

\section{Results}

EMP3 levels in primary breast carcinoma tissues are upregulated compared with adjacent normal tissues. Paired primary breast carcinoma tissues $(n=6)$ were analyzed using RT-qPCR and western blotting to detect the expression status of EMP3. As shown in Fig. 1, the mRNA and protein expression levels of EMP3 were increased, compared with the adjacent non-tumor tissues. These data suggested that EMP3 was upregulated in primary breast carcinoma, which implied that EMP3 may have a tumor oncogenic role in the development of primary breast carcinoma. These results were in agreement with those of a previous study (11).

miR-765 targets the EMP3 transcript 3'UTR and downregulates its expression. In the present study, to examine the regulatory mechanism of EMP3 in primary breast carcinoma, miRwalk was used for miRNA prediction. As shown in Fig. 2A, four candidate miRNAs were identified, including miR-744, miR-486, miR-663 and miR-765. To determine which miRNA was the most effective in regulating EMP3, a luciferase reporter assay was performed. The SK-BR-3 cells were transfected with reporter vector along with the four miRNA mimics. The results demonstrated that miR-765 significantly downregulated the luciferase intensity of pGL3/Luciferase-EMP3-3'-UTR (Fig. 2B), therefore, miR-765, which has been reported to act as a key mediator for the inhibition of growth, migration and invasion in fulvestrant-treated prostate cancer, was selected for further investigation (10). To further determine whether miR-765 directly regulates EMP3, another luciferase-reporter assay was performed to validate 
A

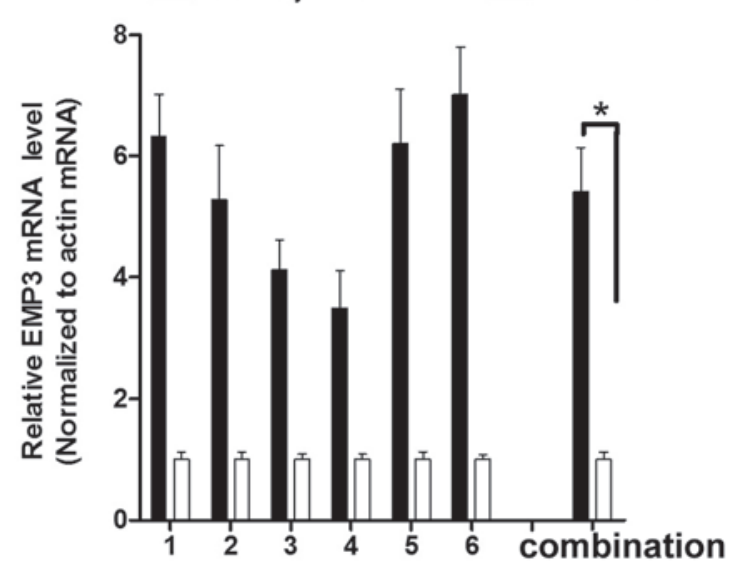

B

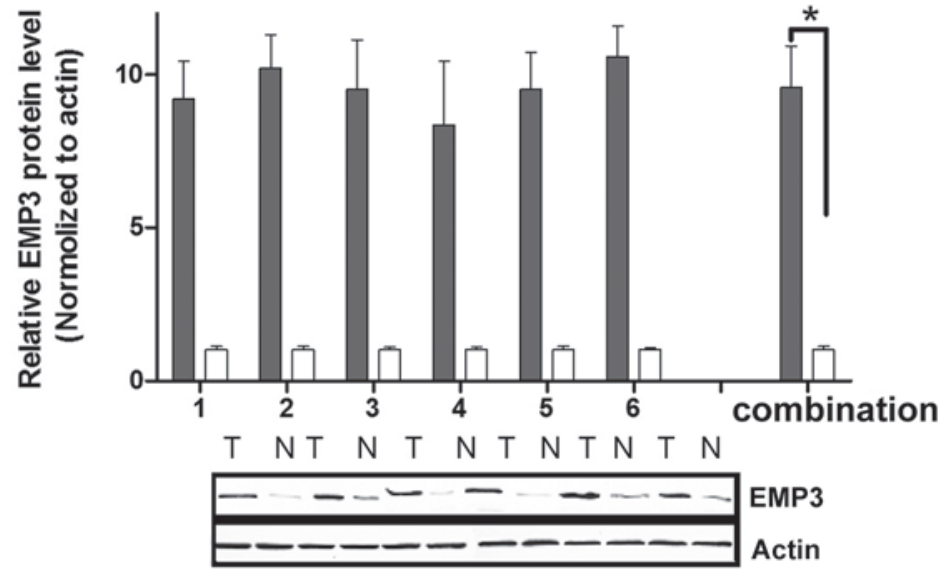

Figure 1. Differential expression of EMP3 in primary breast carcinoma tissues, compared with adjacent normal tissues. (A) Relative mRNA expression levels of EMP3 in six breast cancer tissues and paired adjacent normal tissues. The mRNA levels were detected using reverse-transcription-quantitative polymerase chain reaction. Combination analysis represents the mean EMP3 level in the six samples. (B) Protein expression levels of EMP3 in primary breast tissue and normal tissues using western blot analysis. All data are expressed as the mean \pm standard deviation of three independent experiments. ${ }^{*} \mathrm{P}<0.05$, vs. control. EMP3, epithelial membrane protein 3; T, tumor; N, normal.

the target site in the EMP3-3'UTR (Fig. 2C). The SK-BR-3 cells were transfected with the reporter vector, along with the miR-765 mimics or control. Notably, the miR-765 mimics significantly reduced the luciferase intensity of SK-BR-3 cells transfected with the EMP3-3'UTR reporter vector, but did not affect the luciferase intensity of SK-BR-3 cells transfected with the EMP3-3'UTR mutated vector (Fig. 2C). These results indicated that EMP3 was a direct target of miR-765. In addition, the effect of miR-765 on the expression of endogenous EMP3 mRNA was examined using RT-qPCR. The mRNA level of EMP3 increased 1.45-fold in the SK-BR-3 cells transfected with the miR-765 inhibitor mimics when compared with the control group (Fig. 2D). In addition, the protein level of EMP3 was reduced by 55\% in the SK-BR-3 cells transfected with miR-765 mimics when compared with the control group (Fig. 2D). These results indicated that miR-765 targeted EMP3 and repressed the expression of EMP3.

miR-765 is downregulated in primary breast carcinoma tissues. Based on the data that miR-765 directly targeted EMP3, and that EMP3 was upregulated in primary carcinoma tissues, the miR-765 expression levels were compared using RT-qPCR between six paired primary breast carcinoma tissues and corresponding non-cancerous tissues (Fig. 3A). It was revealed that miR-765 had low levels of expression in all six primary breast carcinoma tissues, and the average level was $\sim 0.5$ times lower than in the corresponding non-cancerous tissues. This suggested that the low expression level of miR-765 was correlated with the high expression level of EMP3. To further confirm the association between miR-765 and EMP3, four breast cancer cell lines were used for the detection of the expression levels of miR-765 and EMP3. As shown in Fig. 3B, miR-765 was inversely correlated with EMP3, which was the same association observed within the clinical specimen.

Knockdown of EMP3 has similar effects to miR-765 on $S K-B R-3$ cells. To further confirm that the effects of miR-765 on the proliferation of SK-BR-3 cells were, at least in part, mediated by EMP3, a shRNA vector was prepared in order to reduce the expression of EMP3. Firstly, western blotting (Fig. 4A) demonstrated that miR-765 and shRNA EMP3 effectively reduced the expression of EMP3 in the SK-BR-3 cells. MTT (Fig. 4B) and Transwell (Fig. 4C-D) assays further confirmed that miR-765 and shRNA EMP3 suppressed the cell viability, and the invasion and migration abilities of the SK-BR-3 cells.

\section{Discussion}

During the past two decades, breast cancer has remained the most common type of cancer in females in China (12). Breast cancer-associated mortality has reduced due to the development of novel diagnostic approaches, drugs and increased understanding of the molecular pathology of this disease (13). However, the toxicity associated with these therapies is significant and a significant obstacle in cancer treatment. Therefore, improvements in developing novel, non-toxic methods for the treatment of patients with breast cancer is required (14). Gene targeted therapy is a relatively recent development, and the identification of more breast carcinoma gene target sites is required. EMP3 has been reported to be a important gene in several types of cancer. Notably, a previous report indicated the decreased expression of EMP3 by promoter methylation is associated with lung cancer (15), and the promoter methylation and mRNA expression levels of EMP3 and PCDH- $\gamma$-A11 have been reported to be closely associated with the malignant development of glioma (16). These reports suggest that EMP3 acts as a tumor suppressor. However, a study reported that EMP3 overexpression in breast carcinoma is significantly associated with histological grade III, lymph node metastasis and marked expression of Her-2 (11). In addition, EMP3 overexpression is associated with oligodendroglial tumors (17), further demonstrating that EMP3 functions as an oncogene. In the present study, the expression level of EMP3 was detected in primary breast carcinoma and compared with that in adjacent noncancerous tissues. The levels of EMP3 were observed to increase 
A

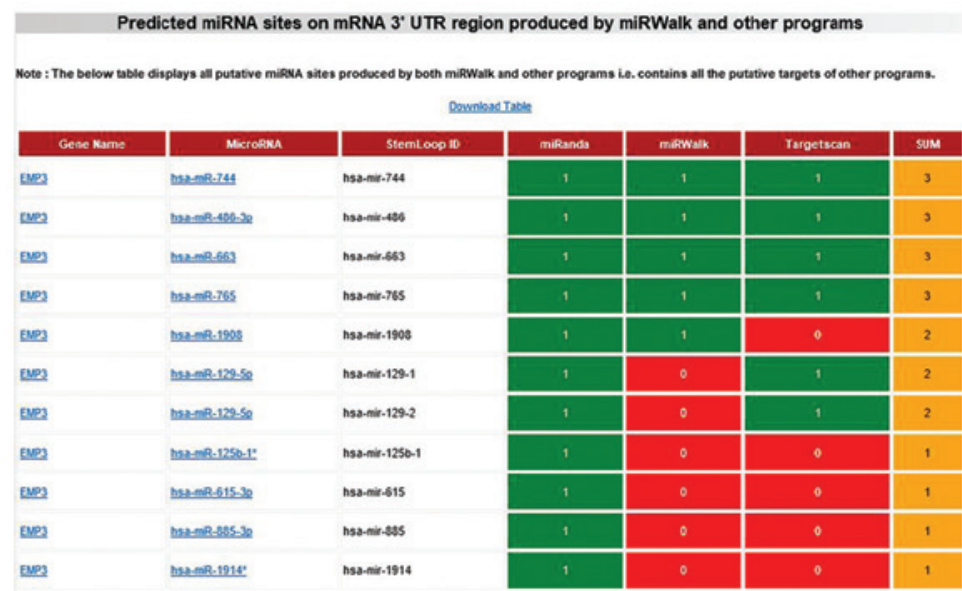

B

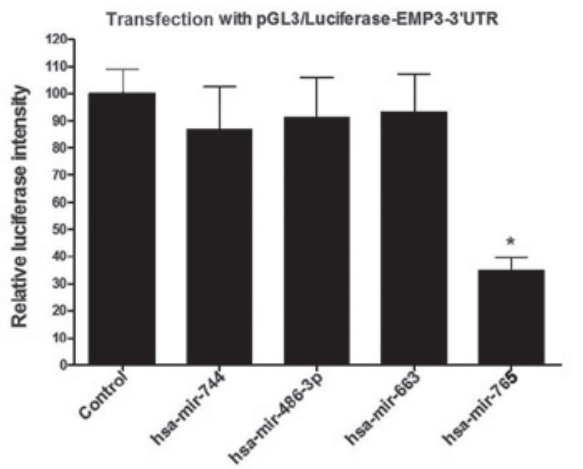

C

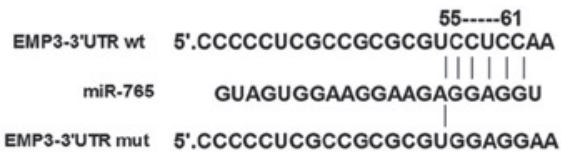

D

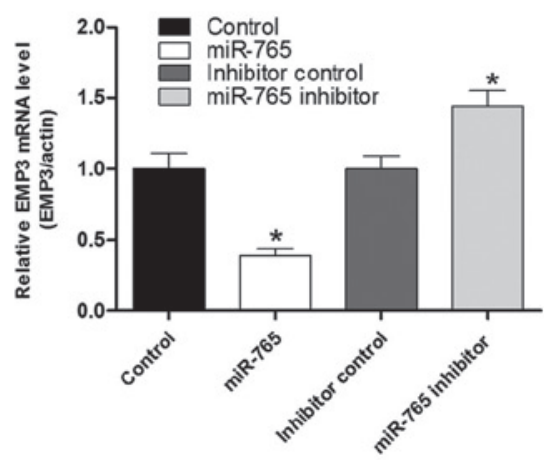

Figure 2. miR-765 directly targets EMP3 and represses mRNA levels of EMP3 in SK-BR-3 cells. (A) Schematic representation of the miRNA target prediction algorithm (miRwalk) screening of the potential miRNAs targeting the EMP3 mRNA 3'UTR. (B) Luciferase constructs were co-transfected with the luciferase construct (pGL3/luciferase EMP3-3'UTR) and five miR mimics, including a control mimic. Luciferase activity was determined $48 \mathrm{~h}$ after transfection, and normalized to the control. (C) Luciferase constructs were transfected into cells transduced with the miR-765 mimics and control. Luciferase activity was determined $48 \mathrm{~h}$ after transfection. The ratio of normalized to control luciferase activity is presented. (D) miR-765 and miR-765 inhibitor were transfected into SK-BR-3 cells and the mRNA level of EMP3 was measured. Actin was used as a loading control. The level of EMP3 in the control and inhibitor control group were set as 1.0. All the data are expressed as the mean \pm standard deviation of three independent experiments. "P $<0.05$, vs. control. miR-765, microRNA-765; EMP3, epithelial membrane protein 3; UTR, untranslated region; WT, wild-type; Mut, mutant.

A

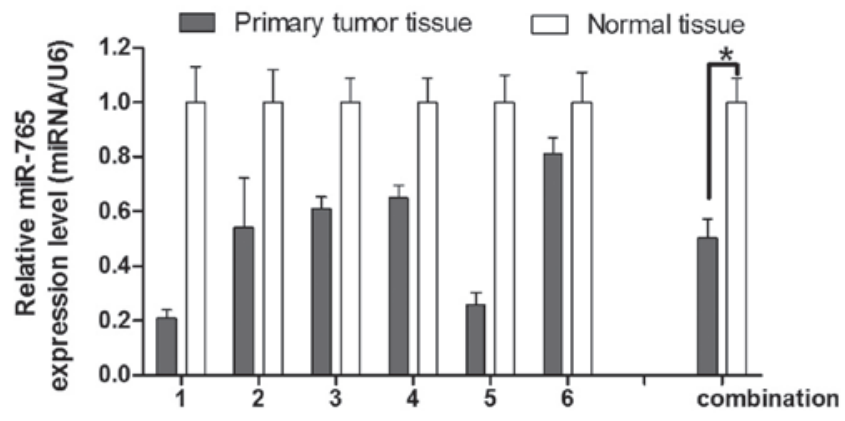

B

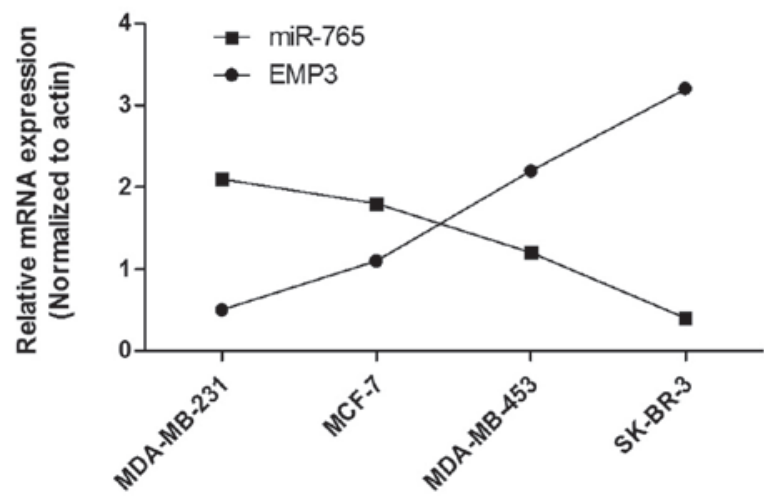

Figure 3. Determination of the expression of miR-765 in primary breast tissues and cell lines. (A) Relative expression levels of miR-765 in primary breast carcinoma tissues, compared with corresponding non-tumor lung tissues. The expression of miR-765 was normalized to U6. (B) Relative expression levels of miR-765 was inversely associated with EMP3 in four breast cancer cell lines (MDA-MB-231, MCF-7, MDA-MB-453 and SK-BR-3). miRNA/miR, microRNA; EMP3, epithelial membrane protein 3.

in the tumor tissues, which was consistent with the results of a study by Zhou et al (11). Subsequent functional investigation using EMP3 shRNA in breast cancer cells also indicated an oncogenic role of EMP3 in primary breast carcinoma.
The promoter methylation of EMP3 is reported to be involved in the regulation of EMP3 in several types of cancer $(16,18)$. However, Zhou et al (11) reported no significant correlation between the methylation status and mRNA 
A

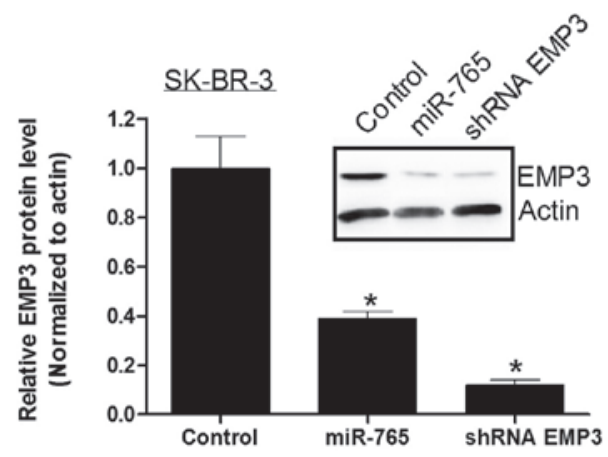

C

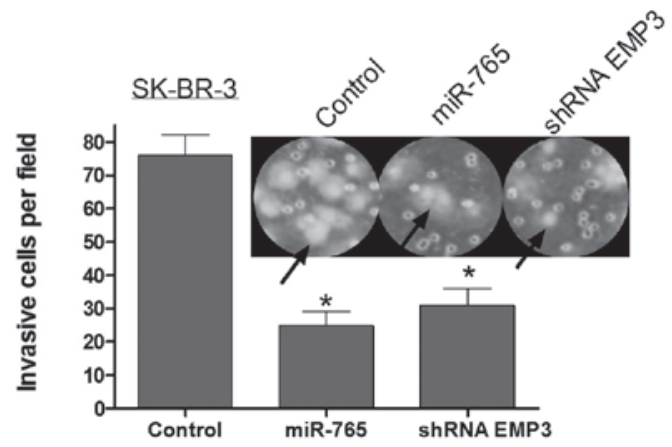

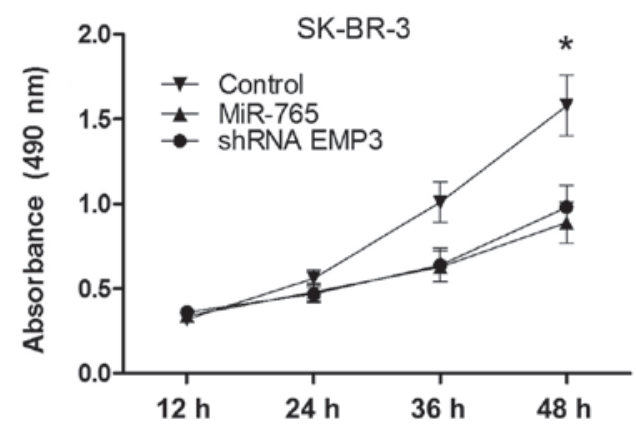

D

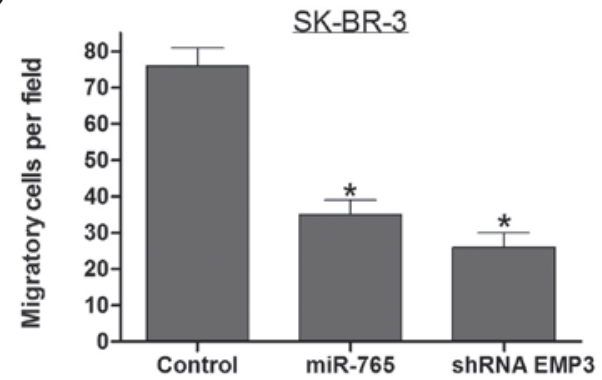

Figure 4. EMP3 modulation accounts for the anti-proliferative effects of miR-765 in SK-BR-3 cells. (A) Western blotting for the protein levels of EMP3 in SK-BR-3 cells following $48 \mathrm{~h}$ of miR-765 and EMP3 shRNA transfection. (B) Effects of miR-765 and shRNA EMP3 on the proliferation of SK-BR-3 cells. (C) Effect of miR-765 and shRNA EMP3 on the cell invasive ability of SK-BR-3 cells. The arrows indicate invaded cells. Scale bar=20 $\mu \mathrm{m}$. (D) Effect of miR-765 and shRNA EMP3 on the migratory ability of SK-BR-3 cells. All data are expressed as the mean \pm standard deviation of three independent experiments. "P<0.05, vs. control. EMP3, epithelial membrane protein 3; miR, microRNA; shRNA, short hairpin RNA.

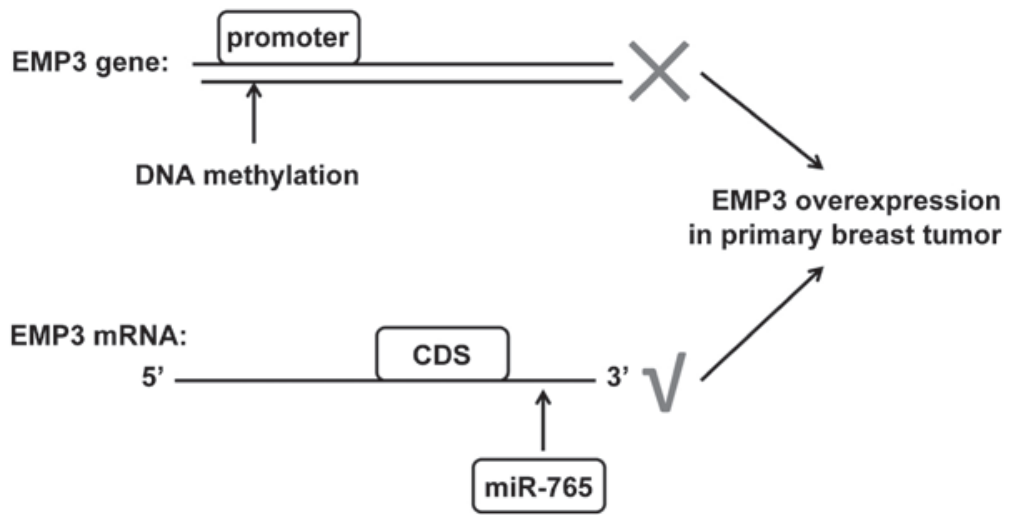

Figure 5. Schematic representation of the hypothetical molecular mechanism of EMP3 regulation in primary breast carcinoma. Top line: DNA methylation regulation mechanism had no effect on EMP3 upregulation in primary breast carcinoma. Bottom line: miR-765 was involved in EMP3 upregulation in primary breast carcinoma. EMP3, epithelial membrane protein 3; miR, microRNA; CDS, coding sequence.

expression levels of EMP3, and concluded that EMP3 may be a novel marker of tumor aggression, and overexpression of EMP3 in primary breast carcinoma is not associated with DNA methylation. Therefore, the present study hypothesized that there are additional factors involved in the regulation of EMP3 in primary breast carcinoma. Post-transcriptional regulation is an important factor in gene regulation, and miRNAs have been reported to be post-transcriptional regulators in a variety of pathogenic conditions, including cancer $(19,20)$. In the present study, miR-765, was identified to directly target EMP3 and be involved in the regulation of EMP3 in breast carcinoma.

Few studies have investigated the role of miR-765, which is located in 1q23.1, in cancer. Leung et al (10) identified a novel fulvestrant signaling cascade involving the ERb-mediated transcriptional upregulation of hsa-miR-765, which suppresses the protein expression of HMGA1 as part of the mechanism underlying the tumor suppressor action of fulvestrant in prostate cancer. In the present study, miR-765 was observed to directly target EMP3 and regulate its expression in breast carcinoma. In addition, MTT and Transwell assays were performed, which indicated that miR-765 suppressed the viability, invasive ability and migratory ability of breast cancer cells. In addition, transfection with EMP3 shRNA yielded results in accordance with the tumor suppressor function of miR-765 on cell viability and invasive ability. The findings of the present study support the presence of an miRNA-induced 
EMP3 regulation mechanism, while the DNA methylation regulatory mechanism had no effect on the upregulation of EMP3 in the primary breast carcinoma (Fig. 5).

In conclusion, the present study revealed three major observations: i) EMP3 was upregulated in primary breast carcinoma tissues, compared with adjacent non-cancerous tissues at the mRNA and protein levels; ii) For the first time, to the best of our knowledge, a specific miRNA, miR-765, was identified to directly target EMP3 and reduce the expression of EMP3 in primary breast carcinoma SK-BR-3 cells; iii) Overexpression of miR-765 or knockdown of the expression of EMP3 resulted in marked inhibition of proliferation and cell invasion in SK-BR-3 cells. These results suggested that EMP3 is upregulated in primary breast carcinoma, functions as an oncogene and is regulated by miR-765. This provides novel insight into the mechanism underlying the miR-765/EMP3 pathway in primary breast carcinoma.

\section{Acknowledgements}

The present study was supported by the Youth Fund of Nantong, Jiangsu Province (grant. no. WQ2014059).

\section{References}

1. Ben-Porath I and Benvenisty $\mathrm{N}$ : Characterization of a tumor-associated gene, a member of a novel family of genes encoding membrane glycoproteins. Gene 183: 69-75, 1996.

2. Taylor V and Suter U: Epithelial membrane protein-2 and epithelial membrane protein-3: Two novel members of the peripheral myelin protein 22 gene family. Gene 175: 115-120, 1996.

3. Ben-Porath I, Kozak CA and Benvenisty N: Chromosomal mapping of Tmp (Emp1), Xmp (Emp2) and Ymp (Emp3), genes encoding membrane proteins related to Pmp22. Genomics 49: 443-447, 1998.

4. Schiemann S, Ruckels M, Engelholm LH, Schwirzke M, Brünner $\mathrm{N}$ and Weidle UH: Differential gene expression in human mammary carcinoma cells: Identification of a new member of a receptor family. Anticancer Res 17: 13-20, 1997.

5. Mackay A, Jones C, Dexter T, Silva RL, Bulmer K, Jones A, Simpson P, Harris RA, Jat PS, Neville AM, et al: cDNA microarray analysis of genes associated with ERBB2 (HER2/neu) overexpression in human mammary luminal epithelial cells. Oncogene 22: 2680-2688, 2003.
6. Evtimova V, Zeillinger R and Weidle UH: Identification of genes associated with the invasive status of human mammary carcinoma cell lines by transcriptional profiling. Tumour Biol 24: 189-198, 2003.

7. Hutvagner G and Zamore PD: A microRNA in a multiple-turnover RNAi enzyme complex. Science 297: 2056-2060, 2002.

8. Ouyang M, Li Y, Ye S, Ma J, Lu L, Lv W, Chang G, Li X, Li Q Wang S, et al: MicroRNA profiling implies new markers of chemoresistance of triple-negative breast cancer. PLoS One 9: e96228, 2014.

9. Yan LX, Huang XF, Shao Q, Huang MY, Deng L, Wu QL, Zeng YX and Shao JY: MicroRNA miR-21 overexpression in human breast cancer is associated with advanced clinical stage, lymph node metastasis and patient poor prognosis. RNA 14: 2348-2360, 2008.

10. Leung YK, Chan QK, Ng CF, Ma FM, Tse HM, To KF, Maranchie J, Ho SM and Lau KM: Hsa-miRNA-765 as a key mediator for inhibiting growth, migration and invasion in fulvestrant-treated prostate cancer. PLoS One 9: e98037, 2014.

11. Zhou W, Jiang Z, Li X, Xu F, Liu Y, Wen P, Kong L, Hou M and $\mathrm{Yu}$ J: EMP3 overexpression in primary breast carcinomas is not associated with epigenetic aberrations. J Korean Med Sci 24: 97-103, 2009.

12. Wang YC, Wei LJ, Liu JT, Li SX and Wang QS: Comparison of cancer incidence between China and the USA. Cancer Biol Med 9: 128-132, 2012.

13. Tinoco G, Warsch S, Gluck S, Avancha K and Montero AJ: Treating breast cancer in the 21st century: Emerging biological therapies. J Cancer 4: 117-132, 2013.

14. Alphandéry E: Perspectives of breast cancer thermotherapies. J Cancer 5: 472-479, 2014.

15. Xue Q, Zhou Y, Wan C, Lv L, Chen B, Cao X, Ju G, Huang Y, Ni R and Mao G: Epithelial membrane protein 3 is frequently shown as promoter methylation and functions as a tumor suppressor gene in non-small cell lung cancer. Exp Mol Pathol 95: 313-318, 2013.

16. Jiang Z, Zhou W, Li XG, Jiang YQ, Wang L, Wang DH, Wang XY and Li XE: The methylation analysis of EMP3 and PCDH-gamma-A11 gene in human glioma. Zhonghua Wai Ke Za Zhi 48: 300-304, 2010 (In Chinese).

17. Li KK, Pang JC, Chung NY, Ng YL, Chan NH, Zhou L, Poon WS and Ng HK: EMP3 overexpression is associated with oligodendroglial tumors retaining chromosome arms 1p and 19q. Int J Cancer 120: 947-950, 2007.

18. Pasini A, Iorio P, Bianchi E, Cerasoli S, Cremonini AM, Faedi M, Guarnieri C, Guiducci G, Riccioni L, Molinari C, et al: LOH 19q indicates shorter disease progression-free interval in low-grade oligodendrogliomas with EMP3 methylation. Oncol Rep 28: 2271-2277, 2012.

19. Fabbri M: miRNAs as molecular biomarkers of cancer. Expert Rev Mol Diagn 10: 435-444, 2010.

20. Leung AK and Sharp PA: MicroRNA functions in stress responses. Mol Cell 40: 205-215, 2010. 